\title{
Characterization of Komagataeibacter isolate reveals new prospects on waste stream valorization for bacterial cellulose production
}

\author{
Pietro Cannazza ${ }^{1 *}$, Antti J Rissanen², Dieval Guizelini ${ }^{3}$, Pauli Losoi², Essi Sarlin², Diego Romano ${ }^{1}$, Ville \\ Santala² and Rahul Mangayil ${ }^{*}$
}

${ }^{1}$ Department of Food, Environmental and Nutritional Sciences (DeFENS), University of Milan, Via Celoria 2, 20133 Milan, Italy

${ }^{2}$ Faculty of Engineering and Natural Sciences, Tampere University, Tampere, Finland

3 Graduate Program in Bioinformatics, Sector of Professional and Technological Education,

Federal University of Parana (UFPR), Curitiba, PR, Brazil

* Correspondence: Author: Pietro Cannazza, Via Celoria 2, Milan, 20133, Italy, Email address: pietro.cannazza@unimi.it; Rahul Mangayil, Korkeakoulunkatu 6, Tampere, 33720, Finland, Email address: rahul.mangayil@tuni.fi

\begin{abstract}
Komagataeibacter spp. have been used for the bioconversion of industrial wastes and lignocellulosic hydrolysates to bacterial cellulose (BC). Recently studies have demonstrated the capacity of Komagataeibacter spp. in the biotransformation of inhibitors found in lignocellulosic hydrolysates, aromatic lignin-derived monomers (LDMs) and acetate. In general, detoxification and BC synthesis from lignocellulosic inhibitors requires a carbon flow from acetyl-coA towards tricarboxylic acid and gluconeogenesis, respectively. However, the related molecular aspects have not yet been identified in Komagataeibacter spp. In this study, we isolated a cellulose producing bacteria capable of synthesizing BC in a minimal medium containing crude glycerol, a by-product from biodiesel production process. The isolate, affiliated to Komagataeibacter genus, synthesized cellulose in minimal medium containing glucose $(3.3 \pm 0.3 \mathrm{~g} / \mathrm{L})$, pure glycerol $(2.2 \pm 0.1 \mathrm{~g} / \mathrm{L})$ and crude glycerol $(2.1 \pm 0.1 \mathrm{~g} / \mathrm{L})$. Genome assembly and annotation identified four copies of bacterial cellulose synthase operon and genes for redirecting the carbon from central metabolic pathway to gluconeogenesis. According to the genome annotations, a BC production route from acetyl-CoA, a central metabolic intermediate, was hypothesized and was validated using acetate. We identified that when $K$. rhaeticus ENS9b was grown in minimal medium supplemented with acetate, BC production was not observed. However, in presence of readily utilizable substrate, such as spent yeast hydrolysate, acetate supplementation improved BC synthesis.
\end{abstract}

Keywords: Komagataeibacter rhaeticus; Bacterial cellulose; Crude glycerol; Minimal medium; Whole-genome analysis; Acetate

\section{Introduction}

Bacterial cellulose (BC), the nanofibrillar form of cellulose, is synthesized by bacteria of diverse genera, among which the most efficient producers are found in the genus Komagataeibacter (formerly Acetobacter and Gluconacetobacter). In general, BC biogenesis is commenced with the substrate synthesis, UDP-glucose, from the glycolytic pathway (via the catalytic 
activities of glucokinase, phosphoglucomutase and UDP-glucose pyrophosphorylase). $\mathrm{Bcs} A, \mathrm{~B} c s \mathrm{~B}, \mathrm{Bcs} \mathrm{D}$, and $\mathrm{B} c s \mathrm{C}$ proteins [encoded by the bacterial cellulose synthase (bcs) operon] catalyse the polymerization of UDP-glucose to $\beta$-1,4-glucan units, transport of the synthesized glucan chain through the periplasmic space, formation of crystalline regions and extracellular export of the synthesized polysaccharide, respectively [1].

$\mathrm{BC}$ is a versatile biopolymer with unique characteristics such as biodegradability, purity (not complexed with lignin, hemicellulose or pectin) and superior material properties. Thus, BC is extensively studied for its use in various fields of applications [2-4]. Despite the versatile characteristics, studies to improve the production metrics and alternative carbon sources for $\mathrm{BC}$ production have been investigated $[5,6]$. To surpass the low production metrics, rational strategies to engineer the cellulose production machinery and optimize the carbon flow through the cellulose synthesis pathway have been conducted in Komagataeibacter spp. [3,7,8]. By employing residual carbon sources from agriculture and industries, researchers have coupled BC production with strategic waste disposal [9-14]. For instance, Wu et al. (2019) reported a BC titer of $\sim 1.5 \mathrm{~g} / \mathrm{L}$ from A. xylinum ATCC 23767 using crude glycerol, a by-product of biodiesel production from kitchen waste [9]. In a study from Leif Jönsson's group, BC production by K. xylinus ATCC 23770 was investigated in a medium containing 3-fold diluted detoxified spruce hydrolysate [11]. The researchers identified that the $\mathrm{BC}$ production was absent in cultivation medium with non-detoxified hydrolysate. Detoxification with activated charcoal removed $\sim 94 \%$ of furan aldehydes [hydroxymethyl furfural (HMF) and furfural] and $\sim 60$ $-70 \%$ of aliphatic acids (acetic acid and formic acid) from the spruce hydrolysate, enabling K. xylinus ATCC 23770 to produce $8.2 \mathrm{~g} / \mathrm{L} \mathrm{BC}$ [11]. However, in these mentioned studies the waste compounds were supplemented to rich growth medium containing other sources of carbon, restricting an exact elucidation of the substrate's contribution towards BC synthesis [15]. Chemically defined media have been used to circumvent the requirement and influence of other carbon containing compounds in growth and $\mathrm{BC}$ production by Komagataeibacter spp. [16-18]. In our recent study, a BC titer of $2.9 \pm 0.3 \mathrm{~g} / \mathrm{L}$ was obtained from a Komagataeibacter isolate statically grown in MA/9 minimal medium containing crude glycerol [19].

In the present study, we report isolation, biochemical characterization and phylogenetic analysis of a Komagataeibacter strain, isolated from Kombucha SCOBY (symbiotic colony of bacteria and yeast). The study progresses by comparing the strain's BC production metrics from glucose, pure glycerol and crude glycerol supplemented to both complex and minimal media. The genome of the strain was sequenced and assembled, and the genetic insights related to carbohydrate uptake mechanisms, BC biogenesis and gluconeogenesis are reported. Taking the genome insights, carbon redirection from pyruvate metabolism towards $\mathrm{BC}$ production, via acetate supplementation to complex medium and minimal medium, together with baker's yeast hydrolysate was investigated. 


\section{Materials and Methods}

Materials and chemicals

Kombucha SCOBY (symbiotic colony of bacteria and yeast) was obtained from Sri Dhanvanthiri Probiotics Ltd, Kodaikanal, India (True Brew Kombucha tea). Sodium chloride, sodium molybdate, potassium chloride, di sodium hydrogen phosphate, dipotassium hydrogen phosphate, potassium dihydrogen phosphate, calcium carbonate, calcium chloride, magnesium sulphate, disodium hydrogen phosphate, citric acid, bromothymol blue and oxidase test disks (Product no: 70439) were purchased from Merck (Germany). Acetic acid and agar were purchased from Fisher Scientific (UK). Glucose and casein amino acids were purchased from VWR International (Belgium). Tryptone, peptone and yeast extract were from Lab M Limited (UK). Ethanol was from Altia Oyj (Finland). Cycloheximide (Product no: C7698), and cellulase from Trichoderma reesei ATCC 26921 (Product no: C2730) was purchased from Sigma-Aldrich (USA). GeneJET Genomic DNA Purification Kit was purchased from Thermo Scientific (USA). Crude glycerol was generously provided by Perstrop AB (Sweden).

Isolation, culturing and characterization of BC-producing isolates

Cellulose producing bacteria were isolated from Kombucha SCOBY of Indian origin (True Brew Kombucha, Sri Dhanvanthiri Probiotics Ltd, Kodaikanal, India). The SCOBY material was cut into small pieces using sterile scalpel and lysed in $50 \mathrm{ml} 1 \mathrm{X}$ Phosphate Buffered Saline (PBS; g/L, 8 $\mathrm{NaCl}, 0.2 \mathrm{KCl}, 1.44 \mathrm{Na}_{2} \mathrm{HPO}_{4}$, and $0.24 \mathrm{KH}_{2} \mathrm{PO}_{4} ; \mathrm{pH} 7.4$ ) containing $1 \%$ cellulase and incubated overnight $(\mathrm{O} / \mathrm{N})$ at $30^{\circ} \mathrm{C}$ and $180 \mathrm{rpm}$. Cycloheximide $(100 \mathrm{mg} / \mathrm{L})$, a fungicide, was included in the isolation experiments in order to prevent the growth of yeast cells in the SCOBY material. The cells released from SCOBY were centrifuged at $1000 \mathrm{~g}$ for 10 minutes at $4^{\circ} \mathrm{C}$, washed thrice with sterile PBS, resuspended and serially diluted in the buffer. The presence of acetic acid bacteria was verified by plating aliquots on to Glucose-Yeast Extract-Calcium carbonate agar (GYC; g/L, 40 glucose, 10 yeast extract, 30 $\mathrm{CaCO}_{3}$ and 15 agar) containing $100 \mathrm{~g} / \mathrm{L}$ cycloheximide. Colonies around the $\mathrm{CaCO}_{3}$ solubilization zones were individually picked, streaked on HestrinSchramm agar (HS-glucose agar; g/L, 5 peptone, 5 yeast extract, $2.7 \mathrm{Na}_{2} \mathrm{HPO}_{4}$, 1.15 citric acid and 15 agar) containing glucose $(20 \mathrm{~g} / \mathrm{L})$ and cycloheximide $(100 \mathrm{~g} / \mathrm{L})$ and incubated at $30^{\circ} \mathrm{C}$ for $3-5$ days. Single colonies from HS agar were inoculated to sterile 6-well culture plates (Argos Technologies, ColeParmer, US) containing HS medium and incubated at $30^{\circ} \mathrm{C}$ statically for 5 days. The cellulose pellicles synthesized at the air/liquid interface were subjected to lysis in $5 \mathrm{ml}$ HS-glucose medium containing $0.5 \%$ cellulase and $100 \mathrm{mg} / \mathrm{L}$ cycloheximide. The cell suspensions were washed in PBS and restreaked on HS-glucose agar. Single colonies were further picked and the enrichment was iterated for two more rounds. BC production in HS-glucose medium, colony and cell morphologies were routinely inspected in between each enrichment step. The isolated colonies were preserved by resuspending the cells in $25 \%$ glycerol stocks and storing in $-80^{\circ} \mathrm{C}$ freezer.

For biochemical characterization tests, the cells released from the $\mathrm{BC}$ pellicle, synthesized from static cultivation of the glycerol stock in HSglucose medium, were used as the pre-inoculum. The characterization tests 
were conducted in MA/9 minimal medium [20] and peptone-yeast extract medium (PY; g/L, 3 peptone and 2 yeast extract) [21]. Growth only in the presence of $30 \%$ glucose, $0.35 \%$ acetic acid, $3 \%$ ethanol, or $3 \%$ ethanol with $4 \%$ acetic acid, as well as acetate and lactate oxidation tests, and catalase test were conducted as described in [21]. Presence of oxidase was tested using oxidase test disks as per manufacturer's instructions.

For strain identification by $16 \mathrm{~S}$ rRNA gene sequencing, the genomic DNA (gDNA) was prepared, from the cells released from the BC pellicle, using GeneJET Genomic DNA Purification Kit as per manufacturer's instructions. Using the identification service offered by Macrogen (Netherlands), the 16S rRNA gene was amplified from the gDNA using primers 27F (AGAGTTTGATCMTGGCTCAG) and 1492R (TACGGYTACCTTGTTACGACTT) and sequenced with primer pairs 785F (GGATTAGATACCCTGGTA) and 907R (CCGTCAATTCMTTTRAGTTT). The 16S rRNA gene sequence can be found in the NCBI GenBank database under the accession number MT093993. Homology comparisons of the 16S rRNA gene were conducted using the nucleotide BLAST tool [22] against the NCBI GenBank 16S rRNA gene sequence repository for Komagataeibacter (taxid:1434011). Multiple sequence alignment and evolutionary analysis against the 16S rRNA gene sequences of Komagataeibacter type strains were conducted using ClustalW [23] and MEGA X using the Neighbor-Joining method with Kimura 2-parameter model [24], respectively.

\section{$B C$ production}

The BC production tests were conducted in HS and MA/9 minimal medium in 6-well culture plates containing $10 \mathrm{ml}$ of respective growth medium individually supplemented with $2 \%$ glucose, pure glycerol and crude glycerol statically incubated at $30^{\circ} \mathrm{C}$ for 10 days. The tests were conducted in duplicates. A substrate blank (i.e., cultivation without the studied carbon sources) was included to detect the effect of yeast extract and tryptone (in HS medium) and casein amino acids (in MA/9 medium) on BC production. Additionally, non-inoculated growth medium was included as the contamination control.

BC synthesis from acetate was studied by cultivating the cells in $50 \mathrm{ml}$ tubes (Star Lab, Germany) containing $10 \mathrm{ml}$ of either HS, MA/9 and M9 (MA/9 medium devoid of casein amino acids) medium and 10-mM and 50$\mathrm{mM}$ acetate. Influence of other carbon sources on BC synthesis from acetate was tested by supplementing $2 \%$ sodium gluconate or $50 \%$ of baker's yeast hydrolysate (dry yeast purchased from local market) to MA/9 medium containing acetate. The pre-treatment of baker's yeast was performed using the method described in [25]. The tests were conducted in duplicates in 6well culture plates containing $10 \mathrm{ml}$ of culture medium. Growth medium devoid of acetate and cells were used as controls to monitor background BC production and contamination, respectively.

\section{Purification of $B C$ pellicles and dry weight measurements}

The $\mathrm{BC}$ pellicles produced at the air/liquid interface were collected from the cultivation vessel. To eliminate the loosely bound cellulose fibrils, the $\mathrm{BC}$ pellicles were rinsed thoroughly with ultrapure water (Milli-Q, EMD Millipore, Germany). To remove the medium components and bacterial cells 
entrapped within the pellicle, $\mathrm{BC}$ was incubated in $0.5 \mathrm{M} \mathrm{NaOH}$ solution and ultrapure water at $60^{\circ} \mathrm{C} \mathrm{O} / \mathrm{N}$. Following the treatments, the cellulose pellicles were washed thoroughly with ultrapure water until neutral $\mathrm{pH}$ was attained and oven dried $\mathrm{O} / \mathrm{N}$ on pre-weighed $46 \times 46 \times 8 \mathrm{~mm}$ weighing boats (Heathrow Scientific, USA) at $60^{\circ} \mathrm{C}$. For acetate production tests in HS medium, BC pellicles were subjected to drying after collecting from the cultivation medium.

\section{Amino acid utilization test}

For amino acid utilization test, K. rhaeticus ENS9b pre-inoculum were prepared from static cultivation of cells in MA/9 medium containing $2 \%$ glucose. The pre-inoculum was inoculated to 96-well microtiter plate wells (initial OD600nm, 0.1) individually containing 22 L-amino acids (alanine, arginine, asparagine, aspartic acid, cysteine, cystine, glutamic acid, glutamine, glycine, histidine, hydroxyproline, isoleucine, leucine, lysine, methionine, phenyl alanine, proline, serine, threonine, tryptophan, tyrosine and valine) as sole carbon source, with a final concentration of $20 \mathrm{mM}$ (except for cystine and tyrosine for which the concentration was $10 \mathrm{mM}$ due to low solubility) in M9 medium (total volume, 200 $1 /$ well). The tests included triplicates for each amino acid. M9 medium devoid of amino acid supplementation and K. rhaeticus ENS9b cells were included as experimental controls to monitor the background growth and contamination, respectively. The microtiter plates were incubated at $30^{\circ} \mathrm{C}$ for 8 days and the optical density measurements at $600 \mathrm{~nm}\left(\mathrm{OD}_{600 \mathrm{~nm}}\right)$ were taken once every 24 hours.

\section{Analytical techniques}

Biomass was determined as optical density measurements at $600 \mathrm{~nm}$ wavelength (OD $600 \mathrm{~nm}$ ) using a spectrophotometer (Ultraspec 500pro, Amersham Biosciences, UK). Substrate utilization, and liquid end metabolites were analysed using HPLC equipped with $300 \mathrm{~mm} \times 8 \mathrm{~mm}$ Shodex SUGAR column (Phenomenex), SIL-20AC HT autosampler (Shimadzu), RID-10A refractive index detector (Shimadzu), and $0.01 \mathrm{M}$ $\mathrm{H}_{2} \mathrm{SO}_{4}$ as the mobile phase. The HPLC samples were prepared as described in [26] and the concentrations of carbon substrates and liquid fermentation metabolites were analysed using respective external standards. The BC dry weights were measured using analytical balance (ES 220A, Precisa, Switzerland). The carbohydrate content in the baker's yeast hydrolysate was analysed using the phenol-sulfuric acid method described in [27] and glucose standards $(0.1-1 \mathrm{mM})$.

\section{Material characterization}

$B C$ films (oven-dried prepared from MA/9 medium) synthesized from each carbon source are designated as BC-glucose, BC-glycerol, BC-crude glycerol, BC-sHS and BC-YhMA/9. BC surfaces and cross-sections were analyzed using a scanning electron microscope (SEM; Zeiss ULTRAplus, Germany).

The X-ray diffraction patterns of the dried BC films were obtained using an X-ray diffractometer (XRD; Empyrean multipurpose diffractometer, PANalytical B.V, US) as described previously [3]. The degree of crystallinity was defined as the ratio between the sum of the 101, 10-1, and 002 peaks and 
the sum of the aforementioned and the amorphous peak. Peak height method (or Segal method) is the widely accepted method to calculate BC crystallinity indices (CIs) $[5,28]$. However, for uniformity with our previous study $[3,19]$ and comprehensive assessment using both amorphous and crystalline regions, the $\mathrm{CI}$ values in this study were calculated using a peak deconvolution method [29].

The thermal behaviour of the $\mathrm{BC}$ films was determined using Thermogravimetric analyzer (TG 209 F3 Tarsus, Netzsch-Gerätebau GmbH, Germany). The weight loss from duplicate samples (size, 3.5-6.0 mg) were studied under $\mathrm{N}_{2}$ atmosphere in $30{ }^{\circ} \mathrm{C}$ to $600{ }^{\circ} \mathrm{C}$ range at a heating rate of $10{ }^{\circ} \mathrm{C} / \mathrm{min}$.

\section{Genome sequencing, assembly and bioinformatics}

K. rhaeticus ENS9b gDNA was extracted as mentioned previously (section Isolation, culturing and characterization of BC-producing isolates) and sequenced at Novogene Europe (Cambridge, UK) using Illumina Novaseq 6000. The raw reads were trimmed using Trimmomatic [30] and denovo assembled into contigs by SPAdes [31]. The contig scaffolding was conducted by SOPRA scaffold assembly tool at Galaxy Europe web platform (usegalaxy.eu; Galaxy Version 0.1) using the SPAdes contig assembly and the paired end Illumina sequencing data [32]. The minimum contig length used in scaffold assembly was assigned as $200 \mathrm{bp}$. Misassemblies were identified using QUAST (5.0.2) [33]. Contig reordering, missassembly correction and gap filling were conducted using GFinisher with $K$. rhaeticus iGEM genome (GenBank accession number, LT575493.1) as the reference sequence [34] and the genome quality was assessed again using QUAST. The genome was annotated using Prokka [35]. The genome completeness was analyzed using the CheckM plugin in the KBase web platform [36,37]. Functional annotation was conducted through the KEGG Automatic Annotation Server (https://www.genome.jp/kaas-bin/kaas main, accessed on 10.09.2020) [38]. Proteins associated with cellulose synthesis, substrate catabolism, and gluconeogenesis were identified either through manual search within the Prokka annotated GenBank file using Unipro UGENE software (v. 33.0) or from KEGG orthology and the generated pathways [39]. For functional analysis of the annotated proteins and domain predictions, the query amino acid sequences were searched against NCBI's conserved domain database (CDD) and InterProScan signatures [40,41]. Plasmids in the raw sequencing reads and the assembled contigs were identified using plasmidSPAdes [42] and Recycler, respectively [43]. Genome coverage was estimated using Bowtie2 [44]. The origin of replication (oriC) was predicted using Ori-finder tool (http://tubic.org/Ori-Finder/; accessed on 05.08.2020) [45]. For rRNAs and tRNA prediction, a homology search was conducted using RNAmmer [46] and tRNAscan-SE [47] tools at the RNAspace (v1.2.1, rnaspace.org, accessed on 28.08.2020) web environment [48].

The chromosome sequence of K. rhaeticus ENS9b can be found in the NCBI GenBank under the accession number CP061369 and the plasmids at CP061370 (p9b_1), CP061371 (p9b_2), CP061372 (p9b_3), CP061373 (p9b_4) and CP061374 (p9b_5).

\section{Results}


Characterization and classification of the cellulose producing isolate

Colony isolation from $\mathrm{CaCO}_{3}$ solubilized zones and iterated streaking of cells from BC pellicles on HS-glucose agar resulted in the enrichment of small, irregularly edged, white coloured smooth colonies (Fig. S1A). Prolonged incubation resulted in the appearance of a white mass on the colony tip changing the morphology to pulvinated colonies which were difficult to pick using an inoculation loop. The isolate, hereafter designated at ENS9b, appeared as rod-shaped cells either singularly, in pairs or in chains with a cell size of $2.6-4.5 \mu \mathrm{m} * 0.6-0.7 \mu \mathrm{m}$ (Fig. S1B).

Phylogenetic analysis using the ENS9b 16S rRNA gene against the Komagataeibacter type strains positioned the isolate among $K$. rhaeticus with a $99 \%$ similarity towards K. rhaeticus DST GL02 ${ }^{\mathrm{T}}$ (Fig. 1).

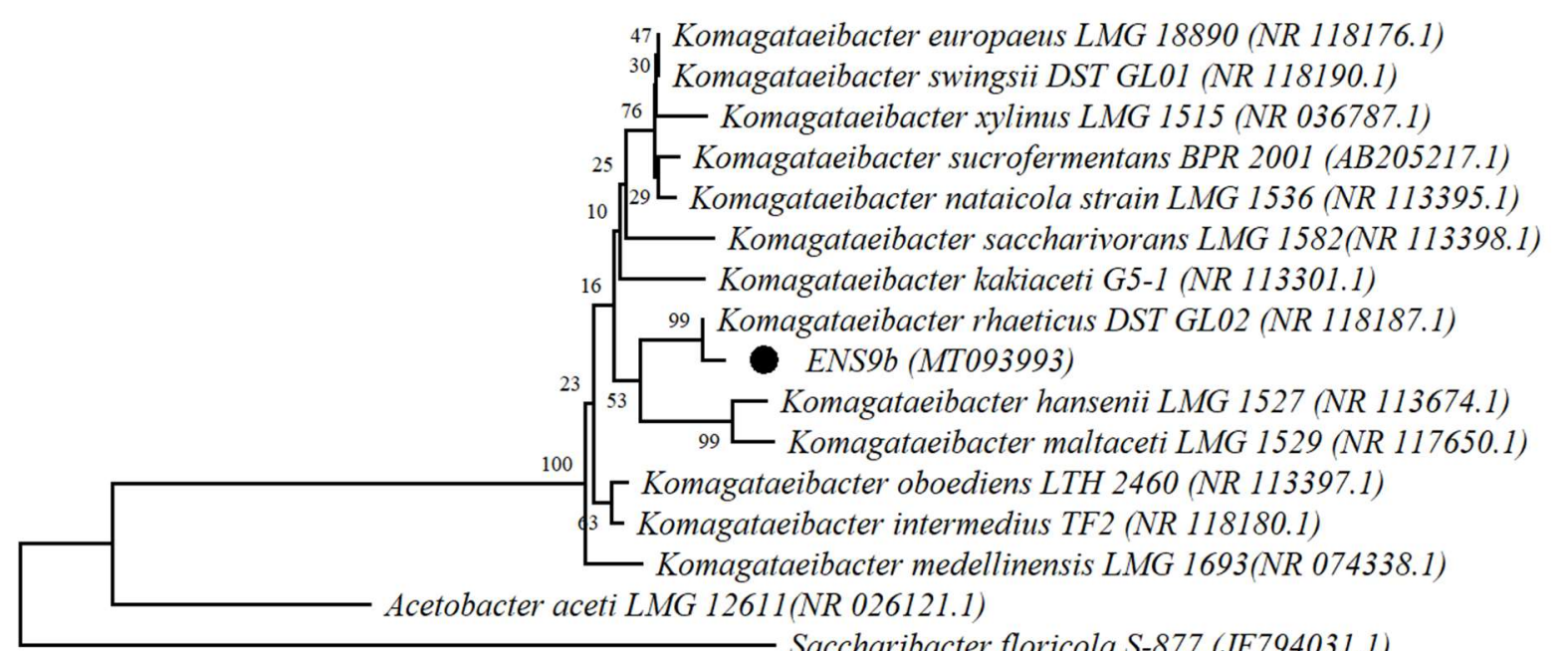

Saccharibacter floricola S-877 (JF794031.1)

0.01

Figure 1. Phylogenetic relationship of ENS9b and Komagataeibacter type strains. The $16 \mathrm{~S}$ rRNA gene tree was rooted using Saccharibacter floricola S-877 and Acetobacter aceti LMG $12611^{\mathrm{T}}$. The position of ENS9b in the phylogenetic tree is highlighted with a dot. NCBI accession numbers are provided in parenthesis.

To restrict the influence of peptone and yeast extract on the isolate's growth, the biochemical characterization tests were initially conducted in MA/9 minimal medium. However, similar to that observed from a related species [19], the isolate grew well with $30 \%$ glucose but poorly in MA/9 medium containing $0.35 \%$ acetic acid, 3\% ethanol, and 3\% ethanol and $4 \%$ acetic acid . Thus, the characterization tests were conducted in PY medium [21]. For cultivations in 3\% ethanol and $4 \%$ acetic acid, the medium $\mathrm{pH}$ remained stable until day 11 with a slight drop thereafter (Fig. S2). Growth in the presence of $0.35 \%$ acetic acid did not reveal any major changes in the medium $\mathrm{pH}$ trend (Fig. S2). However, the capacity of ENS9b to metabolize acetic acid was confirmed by the formation of $\mathrm{BC}$ pellicles and its absence in the control cultivation (PY medium without $0.35 \%$ acetic acid or $3 \%$ ethanol, Fig. S3). Similar to other Komagataeibacter spp., K. rhaeticus ENS9b showed 
positive and negative results for catalase and oxidase tests, respectively, did not require acetic acid for growth, oxidized acetate and lactate in PY medium and demonstrated acetic acid overoxidation (Fig. S4) [49,50].

\section{Bacterial cellulose production in rich and minimal growth medium}

The $\mathrm{BC}$ production and $\mathrm{pH}$ profiles from cultivations in HS medium with respective carbon sources are presented in Figure $2 \mathrm{~A}$. When grown in HS medium, K. rhaeticus ENS9b produced the highest BC titer (and yield) in the medium containing pure glycerol $\left[3.0 \pm 0.1 \mathrm{~g} / \mathrm{L}\right.$ and $\left.1.5 \mathrm{mg} / \mathrm{g}_{\text {substrate }}\right]$, utilizing $55 \pm 0.5 \%$ of the substrate. With crude glycerol as the substrate, the strain utilized $48 \%$ of the carbon source, producing $1.9 \pm 0.2 \mathrm{~g} / \mathrm{L}$ BC $(0.9$ $\mathrm{mg} / \mathrm{g}_{\text {substrate }}$. After the 10-day cultivation period, K. rhaeticus ENS9b completely utilized the supplemented glucose albeit producing only $0.6 \pm 0.0$ $\mathrm{g} / \mathrm{L} \mathrm{BC}$ and gluconic acid as the main liquid end-metabolite $(14.5 \pm 0.3 \mathrm{~g} / \mathrm{L}$, corresponding to $74 \%$ of the initial glucose concentration). Nevertheless, HS medium containing other carbon compounds, such as yeast extract and tryptone, can contribute towards biomass and background BC production $[15,19]$. In substrate blank cultivation, $K$. rhaeticus ENS9b produced $0.05 \pm 0.0$ g/L BC.

In contrast to HS medium, BC production was not observed from cultivations in MA/9 medium devoid of studied carbon sources (substrate blank). Growth in MA/9-glucose medium improved BC synthesis $(3.3 \pm 0.3 \mathrm{~g} / \mathrm{L}$ and $\left.1.7 \mathrm{mg} / \mathrm{g}_{\text {substrate }}\right)$ and reduced gluconate generation $(7.2 \pm 0.2 \mathrm{~g} / \mathrm{L}$, corresponding to $35 \%$ of initial substrate concentration). With pure and crude glycerol as the sole carbon sources, K. rhaeticus ENS9b utilized $72 \%$ of the supplemented substrate synthesizing $2.2 \pm 0.1$ and $2.1 \pm 0.1 \mathrm{~g} / \mathrm{L}$ of $B C(\sim 1.0 \mathrm{mg}$ $\left.\mathrm{BC} / \mathrm{g}_{\text {substrate }}\right)$, respectively. 

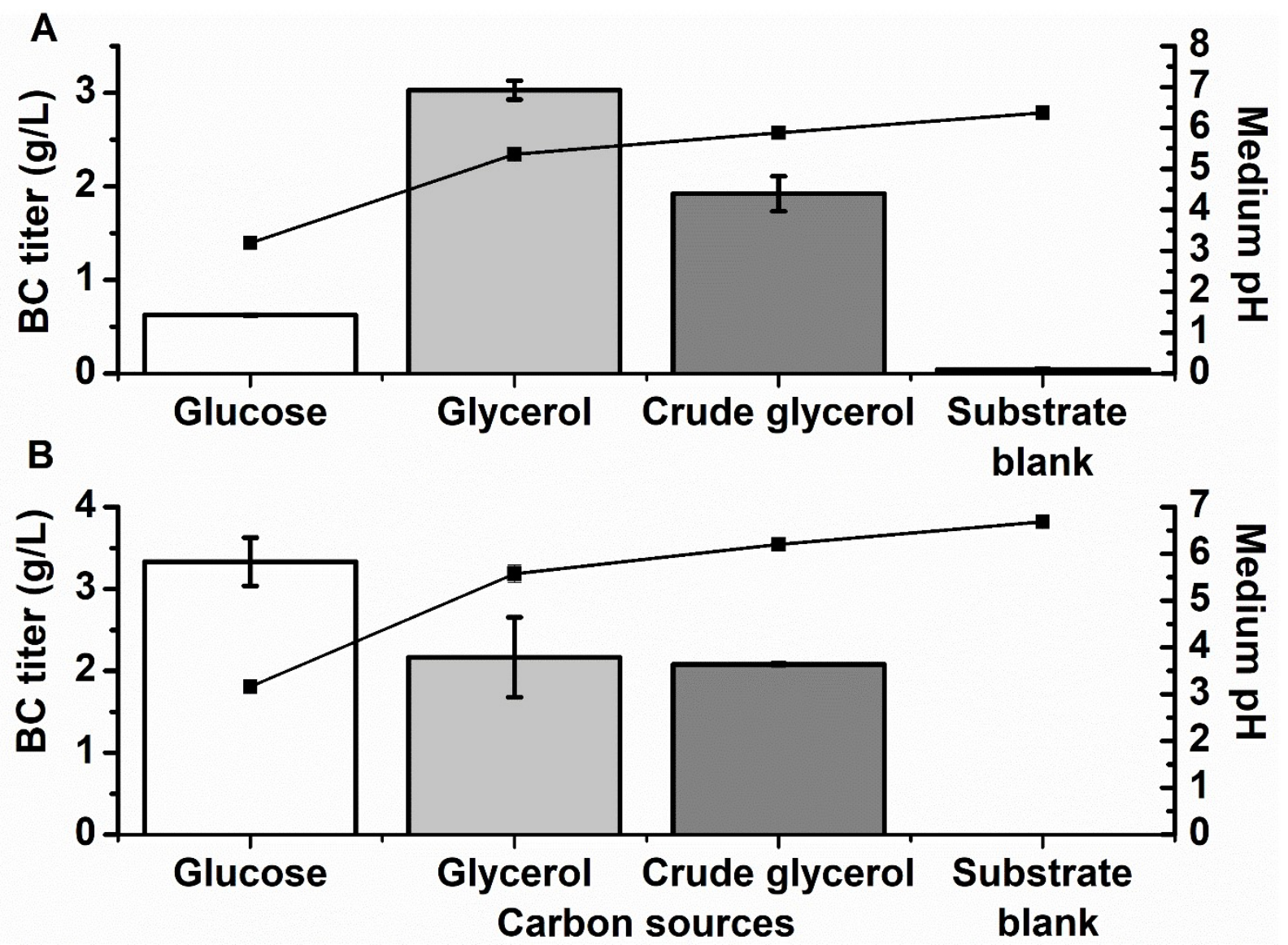

Figure 2. BC production (bars), and $\mathrm{pH}$ (line) plots from static cultivations in (A) HS and (B) MA/9 growth medium supplemented with $2 \%$ glucose, pure glycerol and crude glycerol (MA/9). Substrate blank was included to identify the contribution of medium components (yeast extract and peptone and casein amino acids in HS medium and MA/9 medium, respectively) towards BC production. The data points represent mean experimental results and standard deviations from duplicate cultivations. In some cases, the error bars are smaller than the symbol.

\section{BC film characterization}

The surface and cross-sections as well as the results of XRD and thermogravimetric analyses (TGA) of BC films synthesized by K. rhaeticus ENS9b grown in MA/9 medium with $2 \%$ glucose, pure glycerol and crude glycerol are shown in Figure 3. The surface and cross-sections of BC synthesized from different carbon sources (Fig. 3A) demonstrated the cellulose fibrils to contain the general crisscross network with ordered interconnected layers of $\mathrm{BC}$ pellicles.

The XRD analysis of BC films synthesized by K. rhaeticus ENS9b grown in MA/9 medium with $2 \%$ glucose, pure glycerol and crude glycerol are shown in Figure 3B. Consistent with previous reports [19,51], the diffractograms revealed two dominant peaks between $14.6^{\circ}$ to $17.0^{\circ}$, and between $22.8^{\circ}$ to $25.1^{\circ}$ representing the cellulose I allomorphs I $\alpha$ and I $\beta$, respectively. Though with low intensities and asymmetric shapes, amorphous regions in BC-glucose, BC-pure glycerol and BC-crude glycerol were identified at peaks $21.6^{\circ}, 21.1^{\circ}$ and $20.3^{\circ}$, respectively. The CI values of 
BC films produced from glucose, pure and crude glycerol were $87 \pm 13 \%$, $89 \pm 10 \%$ and $96 \pm 2 \%$, respectively.

The results from thermogravimetric analysis (TGA) of BC films are presented in Figure 3C. The mass losses were observed in three stages. At the first stage $\left(30^{\circ} \mathrm{C}\right.$ to $\left.120^{\circ} \mathrm{C}\right)$, a primary mass loss due to moisture removal was observed as 5\%, 5\% and $4 \%$ for BC-glucose, BC-pure glycerol and BC-crude glycerol, respectively. The second phase, i.e., destruction of crystalline regions and decomposition of the cellulose into glucopyranose monomers, leading to a sharp weight loss was observed at temperatures between $280^{\circ} \mathrm{C}$ - $300^{\circ} \mathrm{C}$. During this phase, BC-glucose, BC-pure glycerol and BC-crude glycerol demonstrated a major mass loss of $59 \%, 64 \%$ and $69 \%$, respectively. Though the mass loss values varied slightly, the residual mass (\%) of the tested BC films remained in the ranges of $27 \%-29 \%$.
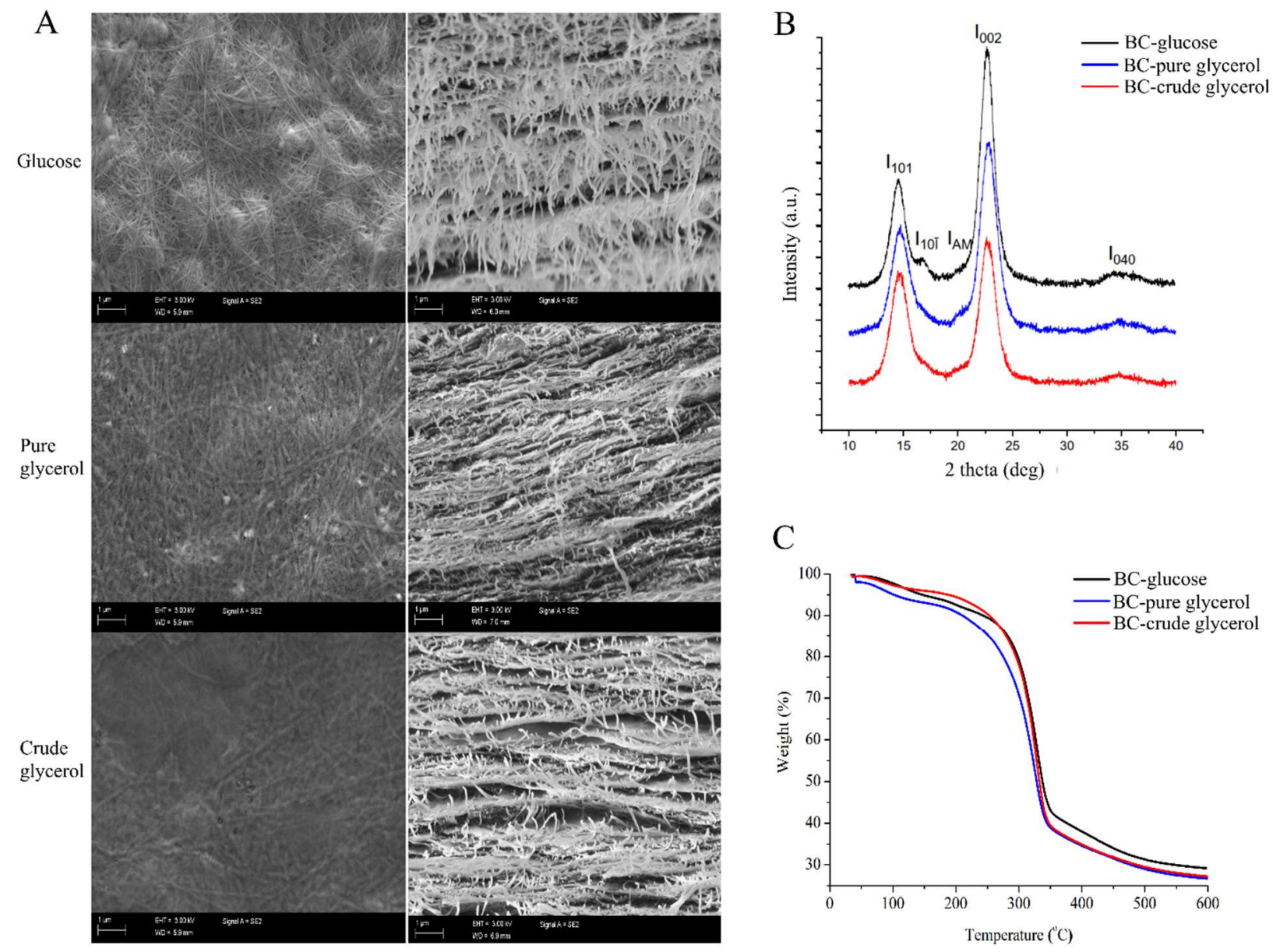

Figure 3. BC film characterization. (A) SEM surface and cross-sectional images, (B) XRD diffractograms and (C) thermogravimetric analysis (TGA) of BC films synthesized from MA/9 medium containing $2 \%$ glucose (BC-glucose), pure glycerol (BC-pure glycerol) and crude glycerol (BC-crude glycerol). The diffraction peaks (subfigure B) at I101 and I10ī, I002 and I040, and IAM represents the crystalline I $\alpha$, I $\beta$ and amorphous regions.

\section{Genome features of K. rhaeticus ENS9b}

The assembly statistics and general features of $K$. rhaeticus ENS9b genome are presented in Table S1. The genome map and functional 
categorization of the gene annotations to KEGG orthology are presented in Figure 4. According to the functional analysis (KEGG classifications and InterProScan), manual search and amino acid alignments (BlastP), and domain predictions (CDD), genes encoding for proteins involved in carbohydrate uptake and metabolism, bacterial cellulose synthesis, and gluconeogenesis from central metabolic pathway were identified from the genome. The genomic positions of genes encoding for the mentioned enzymes and the respective amino acid sequences are presented, in the order, in the Supplementary material.

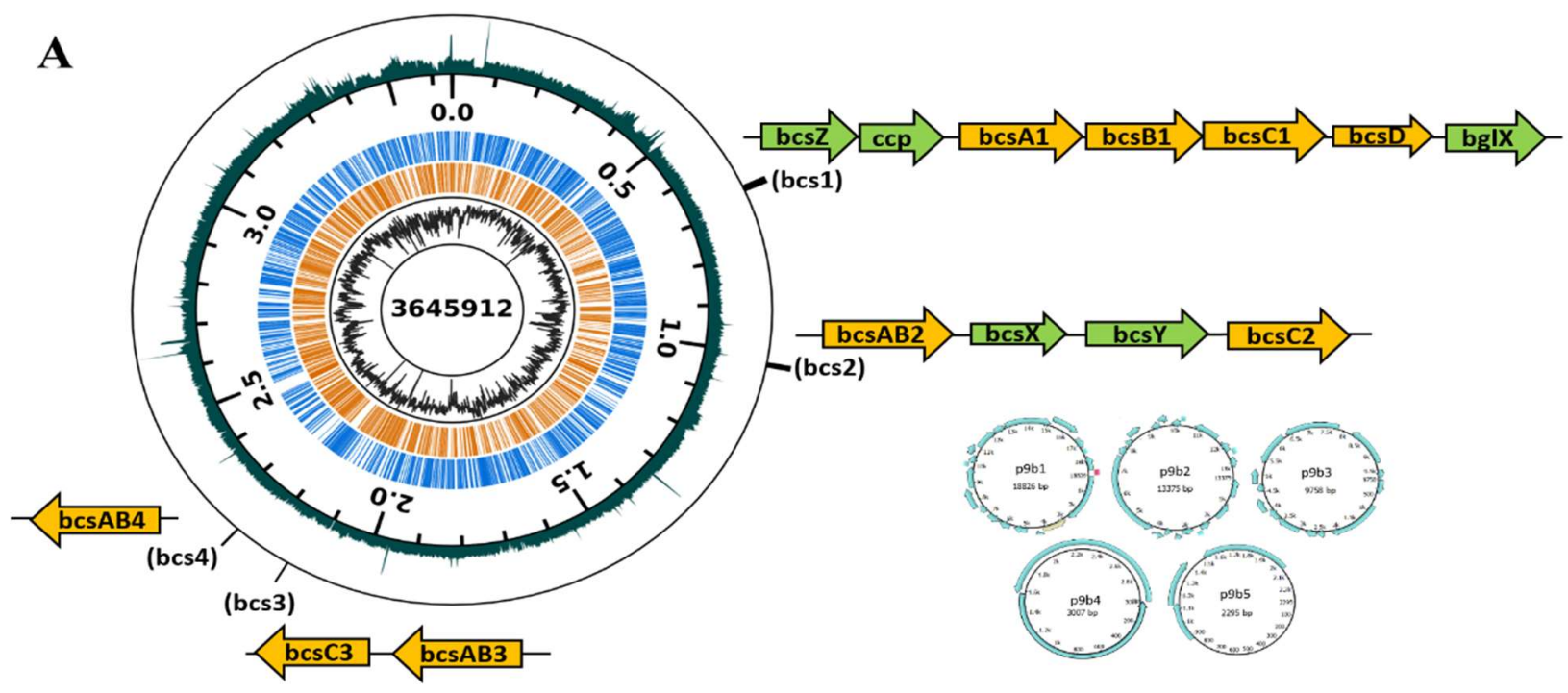

$\mathbf{B}$

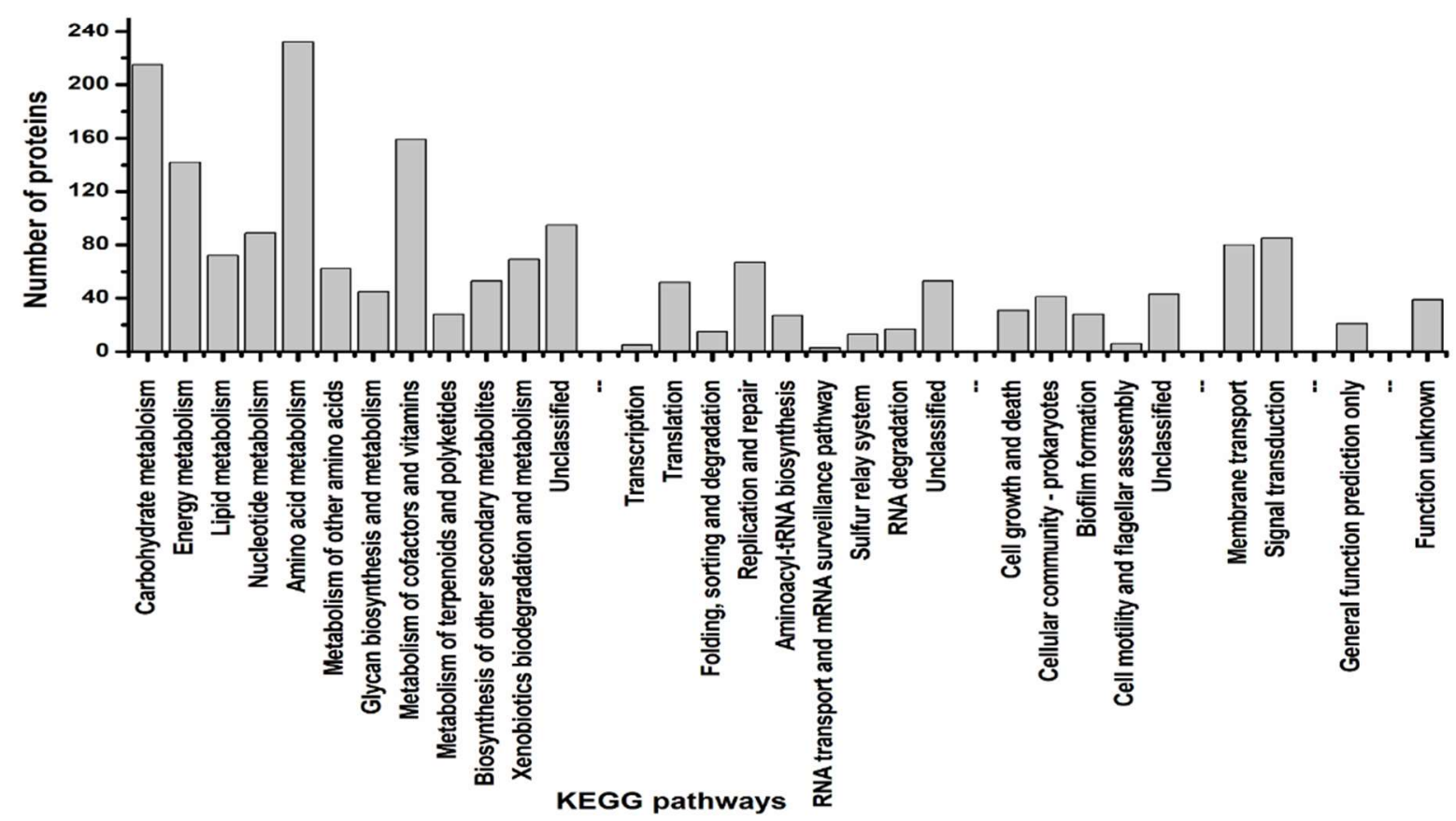

Figure 4. Overview of K. rhaeticus ENS9b genome. (A) Genome map. The K. rhaeticus ENS9b genome totals $3.69 \mathrm{Mbp}$ (GC\% of $63.05 \%$ ), containing 3360 protein-coding gene predictions, 3 rRNAs, 1 tmRNA, 46 tRNAs 
and 3 non coding RNAs. A genome completeness of $100 \%$ was identified from CheckM. The genome consists of a chromosome of $3.65 \mathrm{Mbp}$ (3302 predicted protein-coding regions) and at least 5 plasmids; p9b1 (18.8 $\mathrm{Kbp})$, p9b2 (13.4 Kbp), p9b3 (9.8 Kbp), p9b4 (3 Kbp) and p9b5 (2.3 Kbp) (A inset). Seven contigs (totalling $90 \mathrm{Kbp}$ ) could not be confidently placed in the genome. The displayed data from centre to perimeter are: chromosome size (bp), GC-percentage, CDSs on the reverse strand (in the middle, orange), CDSs on the forward strand (in the middle, blue), chromosome position (major ticks $500 \mathrm{kbp}$, minor ticks $100 \mathrm{kbp}$ ), and coverage (outermost). Both GC-percentage and coverage were calculated with $1 \mathrm{kbp}$ window size. GCpercentage ranged from $33.6 \%$ to $75.5 \%$ and coverage (number of reads mapping to the locus) from 380 to 49600. Coverage is displayed with logarithmic scaling. The bcs operon (orange filled arrows) and the flanking accessory genes (green filled arrows) indicated in the manually curated figures directs to their position in the genome. (B) KEGG classification and functional categorization.

BC biogenesis machineries: K. rhaeticus ENS9b genome contained four copies of bcs operon. The complete bcs operon (bcs1, $9100 \mathrm{bp}$ ) comprising of individual physically adjacent bcsA1, bcsB1, bcsC1 and bcsD genes, in the order, was identified at genomic position 666919:676019 bp. The gene cluster was flanked by accessory genes, bcsZ (genomic position 664722:665759 bp), ccpAx (genomic position 665756:666736 bp) and bglX (genomic position 676251:678452 bp) encoding for $\beta$-1,4-glucanase, cellulose complementing factor protein, and $\beta$-glucosidase, respectively. An additional copy of bglX gene (bglX2) was identified at genomic position 522700:524739 bp. A second bcs operon (bcs2) comprising of a gene fusion encoding for the catalytic and regulatory subunits, bcsAB2, and bcsC2 genes was identified at genomic position 1020008:1030553 bp. The third (at genomic position 2136184:2144443 bp) and fourth (at genomic position 2248494:2253065 bp) bcs clusters were arranged in complement in the genome and comprised of bcsAB3 and bcsC3, and bcsAB4 genes, respectively, and were not flanked with accessory genes associated with $\mathrm{BC}$ assembly/production

Predicted genes involved in carbohydrate uptake and metabolism: In the K. rhaeticus ENS9b genome annotations, 7 genes that encode for putative carbohydrate-selective porins of porin B (OprB) family were identified. Additionally, three copies of aquaporin $\mathrm{Z}$, a major bacterial protein involved in water and glycerol diffusion across the cell membrane was identified from the genome. Similar to other Komagataeibacter spp., an incomplete glycolytic pathway, due to the lack of phosphofructokinase, was observed from $K$. rhaeticus ENS9b genome [52,53]. Glucose oxidation occurs through pentose phosphate pathway (PPP) and Entner - Duodoroff pathway (EDP) via gluconate as the intermediate [catalysed by two quinoprotein glucose dehydrogenases genes $(g d h)$ found at $1924182: 1926572$ and 2594170:2596791 $\mathrm{bp})$. For glycerol metabolism, genes encoding for glycerol uptake facilitator protein (at positions 1285133:1285972 bp), glycerol kinases (1286008:1287507 bp and 1389048:1390547 bp), glycerol dehydrogenases (1201435:1203437 and 1673449:1676036 bp), dihydroxyacetone kinase (1848749:1850380 bp) and triosephosphate isomerase (2116868:2117611 bp) entering into the gluconeogenetic, and central metabolic pathways were identified from the genome.

Putative genes encoding for enzymes involved in redirecting the carbon from Krebs cycle: Indications of BC biogenesis from acetate and putative genes responsible in redirecting the carbon from Krebs cycle towards gluconeogenesis in $K$. rhaeticus isolate was identified in our previous study [19]. Similar to our finding, K. rhaeticus ENS9b genome lacked the genes 
encoding for pyruvate synthase (acetyl-CoA to pyruvate) and phosphoenolpyruvate carboxykinase (oxaloaceticacid to phosphoenolpyruvate). However, the genome contained annotations for genes encoding NAD-dependent malic enzyme (catalysing reversible malate to pyruvate reaction) and pyruvate phosphate dikinase (pyruvate to phosphoenol pyruvate).

\section{$B C$ production from acetate}

To validate the catalytic activities of in silico predicted enzymes involved in gluconeogenesis and corroborate growth and BC production from acetyl$\mathrm{CoA}$, the central compound linking the Krebs and gluconeogenetic pathways, K. rhaeticus ENS9b was grown in presence of acetate.

Acetate utilization and BC production was tested in PY, HS, MA/9 and M9 medium containing 10-mM and 50-mM acetate (Table 1). In PY medium, BC, observed as cellulose fibrils, was produced in both control (devoid of acetate) and sample cultivations. However, due to the absence of an intact pellicle, the fibrils could not be effectively separated from the culture medium for quantification (Fig. S5). At the end of the cultivation in PY-10-mM medium, the strain utilized $78 \pm 5 \%$ acetate. However, growth in PY-50-mM medium resulted in an increase in acetate concentration $(61 \pm 7-\mathrm{mM})$. In the control cultivation without acetate, the strain produced 3.5-mM acetate.

After 14 days of static incubation in HS medium without acetate supplementation, K. rhaeticus ENS9b produced $7 \mathrm{mg}$ of fragile BC pellicles (Fig. S6). As the pellicles were fragile, they were not subjected to alkali and MQ washes, rather oven-dried soon after collecting from the cultivation vessel. Supplementation of $10-\mathrm{mM}$ and $50-\mathrm{mM}$ acetate to HS medium resulted in intact pellicles. In HS medium containing $10-\mathrm{mM}$ and $50-\mathrm{mM}$ acetate, the $K$. rhaeticus cells synthesized $17 \pm 4.5 \mathrm{mg}$ and $20 \pm 0.9 \mathrm{mg} \mathrm{BC}$, respectively (Table 1). The $\mathrm{BC}$ pellicle weights from each replicate cultivations are presented in Fig. S6C. In M9 medium containing 10-mM and 50-mM acetate, cell growth was observed without BC synthesis (Table 1). Similar to M9 medium, BC synthesis was not observed in MA/9 cultivations. Nevertheless, supplementation of casein amino acids in MA/9 medium resulted in $~ 2-9$-fold increase in $\mathrm{OD}_{600 \mathrm{~nm}}$ values. Amino acid utilization test was conducted to study the influence of individual L-amino acids on $K$. rhaeticus growth in M9 medium. The cells could utilize L-alanine, L-arginine, L-asparagine, L-aspartic acid, L-glutamine, L-glutamic acid and L-proline as sole carbon sources for biomass formation (Fig. 5). 


\begin{tabular}{|c|c|c|c|c|}
\hline $\begin{array}{l}\text { Growth } \\
\text { medium }\end{array}$ & $\begin{array}{l}\text { Carbon } \\
\text { containing } \\
\text { compounds }\end{array}$ & $\mathrm{BC}$ production & OD600nm & $\begin{array}{l}\text { Endpoint acetate } \\
\text { concentration }(\mathrm{mM}) \text { and } \\
\text { percentage utilized }(\%)\end{array}$ \\
\hline \multirow[t]{3}{*}{ PY } & $2 \mathrm{~g} / \mathrm{L} \mathrm{YE}$ & $\begin{array}{l}\text { Very loose pellicles. } \\
\text { Could not be } \\
\text { quantified. }\end{array}$ & 1.4 & $3.5-\mathrm{mM}$ \\
\hline & $\begin{array}{l}2 \mathrm{~g} / \mathrm{L} \mathrm{YE}+10-\mathrm{mM} \\
\text { acetate }\end{array}$ & $\begin{array}{l}\text { Loose pellicles. } \\
\text { Could not be } \\
\text { quantified. }\end{array}$ & $1.5 \pm 0.2$ & $1.9 \pm 0.7(78 \pm 5.4 \%)$ \\
\hline & $\begin{array}{l}2 \mathrm{~g} / \mathrm{L} \mathrm{YE}+50-\mathrm{mM} \\
\text { acetate }\end{array}$ & $\begin{array}{l}\text { Loose pellicles. } \\
\text { Could not be } \\
\text { quantified. }\end{array}$ & $1.8 \pm 0.4$ & $\begin{array}{l}61.2 \pm 7.8 \text { (Higher than } \\
\text { initially supplemented) }\end{array}$ \\
\hline \multirow[t]{3}{*}{$\mathrm{HS}^{1}$} & $5 \mathrm{~g} / \mathrm{L} \mathrm{YE}$ & $7 \mathrm{mg}$ & $\begin{array}{l}\text { Cells within the } \\
\text { pellicle. Did not } \\
\text { quantify. }\end{array}$ & ND \\
\hline & $\begin{array}{l}5 \mathrm{~g} / \mathrm{L} \mathrm{YE}+10-\mathrm{mM} \\
\text { acetate }\end{array}$ & $17 \pm 4.5 \mathrm{mg}$ & $\begin{array}{l}\text { Cells within the } \\
\text { pellicle. Did not } \\
\text { quantify. }\end{array}$ & $2.5 \pm 2.0(74 \pm 29.4 \%)$ \\
\hline & $\begin{array}{l}5 \mathrm{~g} / \mathrm{L} \mathrm{YE}+50-\mathrm{mM} \\
\text { acetate }\end{array}$ & $20 \pm 0.9 \mathrm{mg}$ & $\begin{array}{l}\text { Cells within the } \\
\text { pellicle. Did not } \\
\text { quantify. }\end{array}$ & $34.6 \pm 4.9(33 \pm 9.4 \%)$ \\
\hline \multirow[t]{3}{*}{$\mathrm{MA} / 9$} & - & ND & 0.8 & ND \\
\hline & 10-mM acetate & ND & $1.4 \pm 0.2$ & $0.9 \pm 0.0(91.5 \pm 1.5 \%)$ \\
\hline & 50-mM acetate & ND & $1.5 \pm 0.1$ & $7.5 \pm 3.5(79 \pm 7.8 \%)$ \\
\hline \multirow[t]{3}{*}{ M9 } & - & ND & 0.1 & ND \\
\hline & 10-mM acetate & ND & $0.5 \pm 0.1$ & $0.4 \pm 0.0(95.3 \pm 0.2 \%)$ \\
\hline & 50-mM acetate & ND & $0.9 \pm 0.1$ & $2.2 \pm 1.5(98.2 \pm 3.6 \%)$ \\
\hline
\end{tabular}

${ }^{1}$ Due to the fragile nature of the $\mathrm{BC}$ pellicles produced from the control cultivation, the pellicles were not subjected to alkali and MQ wash. The pellicles from the cultivation vessel were collected and oven-dried at $60^{\circ} \mathrm{C}$. For comparison, the pellicles synthesized from HS containing 10-mM and 50-mM acetate were treated similarly. These pellicles can contain medium components that may alter the titers. Thus, the dried weights of untreated pellicles are presented. 


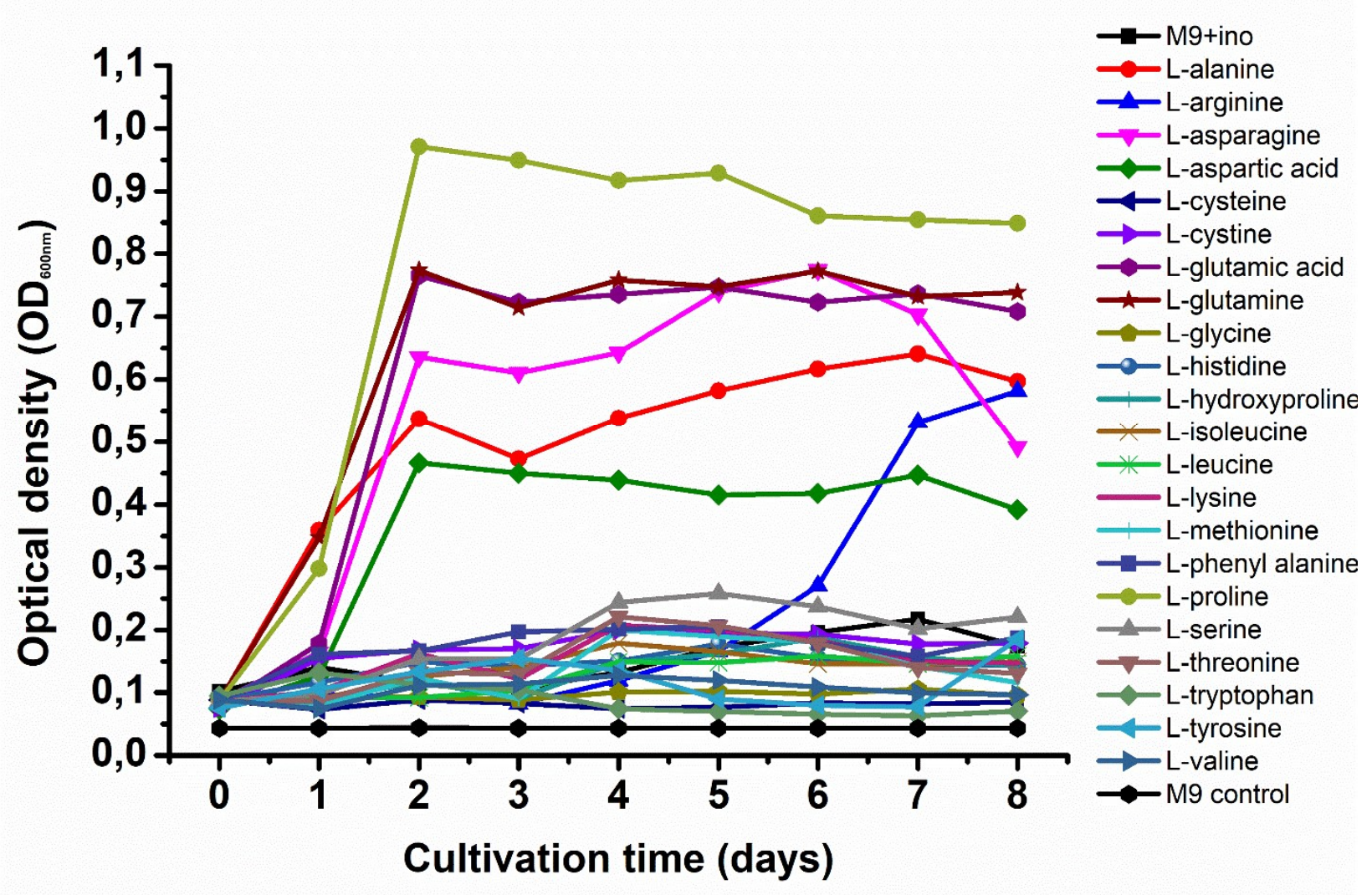

Figure 5. Amino acid utilization profile of K. rhaeticus ENS9b cells grown in M9 medium individually containing $22 \mathrm{~L}$-amino acids. The test was conducted in 96-well microtiter plate wells containing a total volume of $200 \mu \mathrm{l} /$ well. The experimental controls included M9 medium containing cells but devoid of amino acid supplementation (M9+ino control) and M9 medium devoid of cells and amino acids (M9 control). The microtiter plates were incubated at $30^{\circ} \mathrm{C}$ for 8 days and the OD600nm measurements were taken once every 24 hours. The presented data is the averaged value obtained from triplicate cultivations. The error bars are not included for clarity.

In comparison to the control cultivation, an improvement in $\mathrm{BC}$ production was observed from $K$. rhaeticus ENS9b cells grown in HS medium containing acetate. However, in a medium devoid of readily metabolizable substrates the carbon in acetate was directed towards biomass formation (Table 1). Taking these cues, we studied the effect of baker's yeast hydrolysate supplementation in MA/9 medium (YhMA/9) on BC production from acetate [25]. HS and simulated HS media (sHS), i.e., HS medium with $50 \%$ of baker's yeast hydrolysate and $0.2 \%$ casein amino acids to replace the yeast extract and casein amino acids, respectively, were used as the experimental control (Fig. S7 A). Figure 6 presents the BC titers from acetate supplementation to HS, sHS and YhMA/9 media. In the cultivation media devoid of acetate, $K$. rhaeticus ENS9b cells synthesized BC from the control cultivations. In comparison to HS medium, the BC production was higher in sHS and YhMA/9 media attributing it towards the inclusion of 50\% baker's yeast hydrolysate. The phenol-sulfuric acid test indicated a total carbohydrate content in HS (contributed by yeast extract) and sHS (from $50 \%$ of baker's yeast hydrolysate) medium as $7.7-\mathrm{mM}$ and $76-\mathrm{mM}$, respectively. The results 
presented in Fig. 6 clearly distinguishes the improvement in BC production from acetate supplemented media. However, only the cultivations in YhMA/9 - acetate media demonstrated statistically significant $(\mathrm{p}<0.05$, Twosample t-test) titers. Deducting the contribution of yeast extract and baker's yeast hydrolysate in respective medium, BC titers of $15-25 \mathrm{mg} / \mathrm{L}, 75-227$ $\mathrm{mg} / \mathrm{L}$ and $197-327 \mathrm{mg} / \mathrm{L}$ were obtained from acetate supplemented HS, sHS and YhMA/9 media.

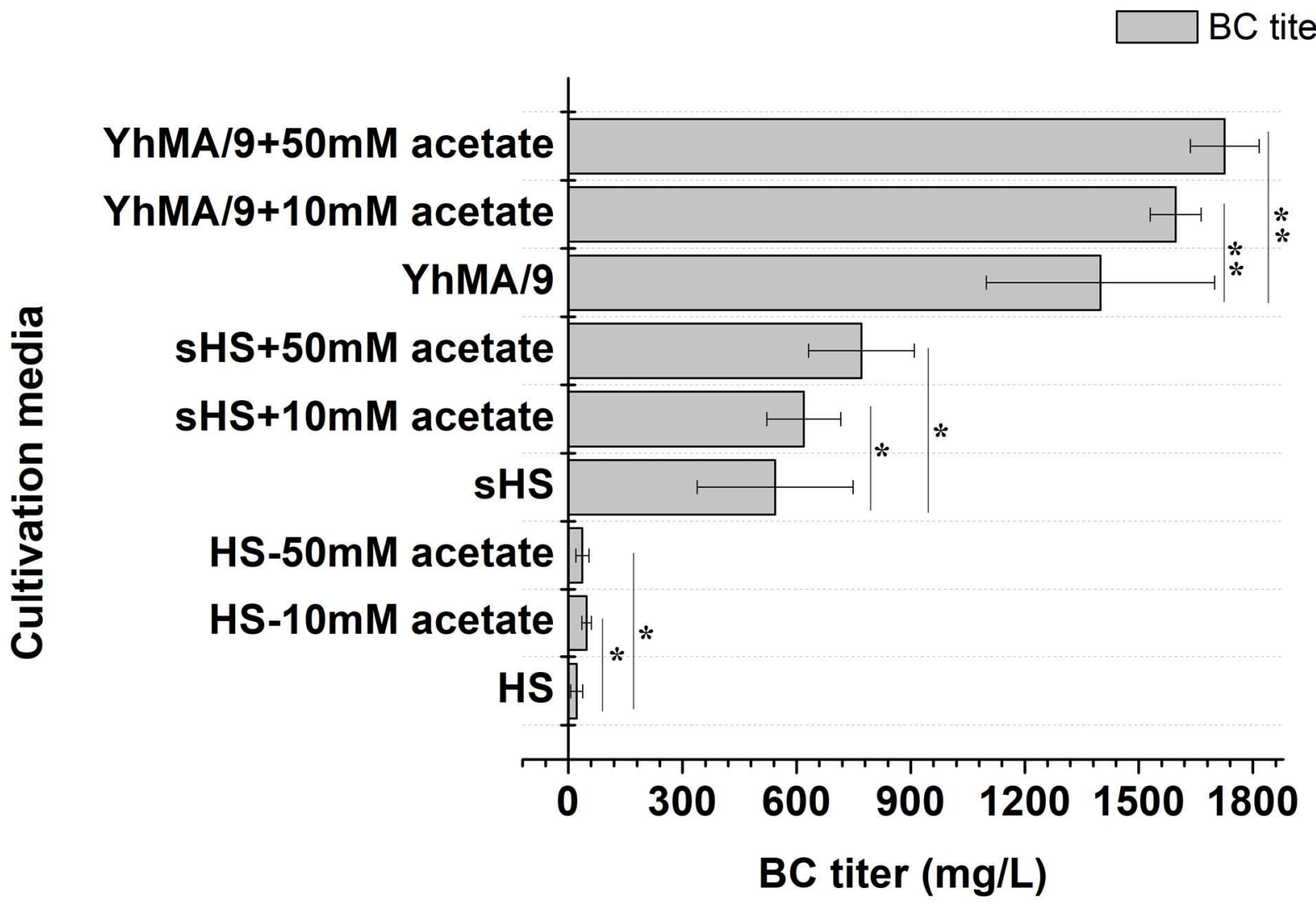

Figure 6. BC production of $K$. rhaeticus ENS9b cells in control and sample cultivations containing 10-mM and 50-mM acetate in HS, sHS and YhMA/9 media. The presented results are the averaged values (duplicates for control cultivations and triplicates for media with acetate) and the error bars represent the standard deviations. Two sample t-test with equal variances using the log10 transformed values were implemented for the comparisons between the control and sample cultivations. Samples with $\mathrm{p}>0.05$ and $\mathrm{p}<0.05$ are represented by ${ }^{*}$ and ${ }^{*}$, respectively.

SEM, XRD and TGA characterization of BC films produced from acetate supplemented sHS and YhMA/9 medium are shown in Fig. S8. Consistent with the prior results (section BC film characterization), peaks representing cellulose I allomorphs (I $\alpha$ and $\mathrm{I} \beta$ ) and amorphous regions were identified at $14.7^{\circ}, 16.9^{\circ}$ and $22.2^{\circ}$, respectively. The peak deconvolution study identified the CI values from BC-sHS and BC-YhMA/9 as $94 \%$ and $92 \%$, respectively. TGA analysis indicates a similar mass loss trend among the BC films, with a residual mass of $\sim 28 \%$. 


\section{Discussion}

$\mathrm{BC}$ is a versatile biopolymer synthesized by bacteria that require mild conditions, simple growth media and can utilize wide ranges of substrates for growth. Komagataeibacter strains have been investigated for their growth and $\mathrm{BC}$ production capacities from conventional sugars, industrial wastes and detoxified lignocellulosic biomass [9,13,19,54-57]. Glucose is an excellent carbon source for BC production, as it is easily transported into the cell and is efficiently incorporated into the cellulose biosynthetic pathway. Komagataeibacter spp. lacks phosphofructokinase incompleting the glycolytic pathway. Glucose oxidation in Komagataeibacter spp. occurs in two routes, via glucose-6-phosphate (entering into PPP) and through gluconate generation which is exported extracellularly (or directly oxidized into the medium by surface exposed gdh). In addition to the drop in the medium $\mathrm{pH}$, gluconate synthesis reduces the glucose availability for BC biosynthesis [3,8,19]. Compared to the HS medium, the improved production titer $(3.3 \pm 0.3 \mathrm{~g} / \mathrm{L} \mathrm{BC})$ and reduced gluconic acid generation $(7.2 \pm 0.2 \mathrm{~g} / \mathrm{L})$ from MA/9 cultivations can be attributed to the buffering capacity in the growth medium [58]. Additionally, the presence of essential macro and micronutrients in the original MA/9 medium composition, may positively contribute in bacterial growth and cellular metabolism [59]. The obtained BC titers from MA/9 medium are comparable to the previously published results from minimal media. For instance, Forng et al. (1989) reported a modest BC titer of $0.1 \mathrm{~g} / \mathrm{L}$ from A. xylinum static cultivations [17]. In another study, de Souza et al. (2019) reported a titer of $0.22 \mathrm{~g}$ BC from K. hansenii ATCC 23769 grown in 10 $\mathrm{ml}$ of minimal medium containing glucose [59]. Recently, using a Komagataeibacter isolate cultivated in similar conditions and $20 \mathrm{~g} / \mathrm{L}$ glucose as the carbon source we reported a titer of $2.2 \pm 0.1 \mathrm{~g} / \mathrm{L} \mathrm{BC}$ [19]. In contrast to glucose cultivations in this study, glycerol as the growth substrate demonstrated an improved substrate utilization (HS medium, $55 \pm 0.5 \%$ and MA/9 medium, $72 \pm 4 \%$ ), albeit with a slight drop in BC production titer (HS medium, 3.0 $\pm 0.1 \mathrm{~g} / \mathrm{L} ; \mathrm{MA} / 9$ medium, $2.2 \pm 0.1 \mathrm{~g} / \mathrm{L} \mathrm{BC}$ ). BC synthesis is regulated by the enzymatic activities of diguanylate cyclase, phosphodiesterase, UDP-glucose pyrophosphorylase, and bcs operon, that are in-turn regulated by the physiological conditions in which the bacterium is cultivated [60]. When Komagataeibacter spp. are grown in minimal medium, the rate-limiting step for $\mathrm{BC}$ biogenesis is partly determined by the carbon source of choice. In case of glycerol, reduced carbon flux towards gluconeogenesis route could be attributed towards the production metrics [61].

In industrial point of view, it is reported that a BC titer of $15 \mathrm{~g} / \mathrm{L}$ in 50 hours (productivity, $0.3 \mathrm{~g} / \mathrm{L} / \mathrm{h}$ ) is required to match to the production efficiency of plants [62]. Thus far, the highest volumetric yield (15 g/L BC in 7 days; productivity $0.1 \mathrm{~g} / \mathrm{L} / \mathrm{h}$ ) has been reported from a Komagataeibacter isolate cultivated in HS medium containing $4 \%$ glucose and $1.4 \%$ ethanol as the inducer [63]. However, utilization of conventional sugars and inducing compounds hinders the production costs and scale-up. Integrating waste valorization with cellulose production is one way to upscale the production processes. Agricultural and industrial waste residues, to name a few, citrus pulp waste, distillery wastewater, crude glycerol, fermentation by-products and detoxified lignocellulosic hydrolysates have been employed as 
alternative carbon and nitrogen sources for $\mathrm{BC}$ production, with titers ranging from $1-13 \mathrm{~g} / \mathrm{L}$ in $2-15$ cultivation days [13,19,64-66]. A recent study reported the production of $12-16 \mathrm{~g} / \mathrm{L}$ BC from lignocellulosic biomass hydrolysates using K. xylinus ATCC 53524 statically grown in a medium containing $50 \mathrm{~g} / \mathrm{L}$ glucose and $20 \mathrm{~g} / \mathrm{L}$ corn steep liquor. Identification of respective enzymes involved in LDM biotransformation could pave way to exciting biotechnological prospects. Nevertheless, for BC synthesis from LDM catabolism, the carbon needs to be redirected from acetyl-coA towards gluconeogenesis. Initial observations of BC formation from acetate were observed from the biochemical characterization tests. In Komagataeibacter spp., acetyl-coA synthetase converts acetate to acetyl-coA, thereby entering into the Krebs cycle. Similarly found in another K. rhaeticus isolate and Escherichia coli genomes, annotations for genes encoding NAD-dependent malic enzyme and pyruvate phosphate dikinase in K. rhaeticus ENS9b genome aiding the hypothesis to route the carbon from Krebs cycle towards gluconeogenesis, was validated using acetate for the proof-of-concept study $[19,67]$. Acetate is generally used as an inducer compound to augment BC production [55,68]. Wang et al., (2018) reported that Komagataeibacter sp. W1 strain could synthesize fragile BC pellicles $(\sim 70 \mathrm{mg})$ from HS medium containing $20 \mathrm{~g} / \mathrm{L}$ of acetate [55]. Our tests identified that availability of other carbon compounds was crucial to promote biomass formation and generate thick BC pellicles. In the absence of readily utilizable carbon, acetate was primarily utilized for biomass formation (Table 1; MA/9 and M9). Inclusion of baker's yeast hydrolysate as an additional carbon source in MA/9 medium improved the BC titer from acetate. Future work will investigate the applicability of spent yeast hydrolysates on BC synthesis, bioprocess optimization using statistical design models and adapted laboratory evolution of the K. rhaeticus strain on LDMs.

Supplementary Materials: The manuscript is accompanied with a supplementary files containing figures, table and amino acid sequences of proteins involved in carbohydrate uptake and metabolism, and BC biogenesis.

Funding: This work was supported by Academy of Finland (Project No. 323214 for R.M.) and Kone Foundation (Project No. 201803224 for A.J.R.). P.C. acknowledges the Erasmus+ program and the doctoral program "Food systems" from the University of Milan for financial support.

Acknowledgments: R.M. thanks Rohith Krishnan for proving the SCOBY sample.

Author Contributions: P.C. and R.M. designed the study and conducted the strain isolation, characterization, and BC production works. R.M., A.J.R., D.G. and P.L. performed the genome assembly and bioinformatics tasks. E.S. conducted the material characterization works. P.C. and R.M. interpreted the data and wrote the original draft. A.J.R., E.S., D.R. and VS reviewed the manuscript. R.M. supervised the work.

\section{References}

1. Römling, U.; Galperin, M.Y. Bacterial cellulose biosynthesis: diversity of operons, subunits, products, and functions. Trends Microbiol. 2015, 23, 545-557, doi:10.1016/j.tim.2015.05.005.

2. Wang, J.; Tavakoli, J.; Tang, Y. Bacterial cellulose production, properties and applications with different culture methods - A review. Carbohydr. Polym. 2019, 219, 63-76, doi:10.1016/j.carbpol.2019.05.008. 
3. Mangayil, R.; Rajala, S.; Pammo, A.; Sarlin, E.; Luo, J.; Santala, V.; Karp, M.; Tuukkanen, S. Engineering and Characterization of Bacterial Nanocellulose Films as Low Cost and Flexible Sensor Material. ACS Appl. Mater. Interfaces 2017, 9, 19048-19056, doi:10.1021/acsami.7b04927.

4. Vuorinen, T.; Laurila, M.-M.; Mangayil, R.; Karp, M.; Mäntysalo, M. High Resolution E-Jet Printed Temperature Sensor on Artificial Skin. In IFMBE Proceedings; 2018; Vol. 65, pp. 839-842 ISBN 9789811051210.

5. Thorat, M.N.; Dastager, S.G. High yield production of cellulose by a Komagataeibacter rhaeticus PG2 strain isolated from pomegranate as a new host. RSC Adv. 2018, 8, 29797-29805, doi:10.1039/C8RA05295F.

6. Dikshit, P.K.; Kim, B.S. Bacterial cellulose production from biodiesel-derived crude glycerol, magnetic functionalization, and its application as carrier for lipase immobilization. Int. J. Biol. Macromol. 2020, doi:10.1016/j.ijbiomac.2020.03.047.

7. Florea, M.; Hagemann, H.; Santosa, G.; Abbott, J.; Micklem, C.N.; Spencer-Milnes, X.; De Arroyo Garcia, L.; Paschou, D.; Lazenbatt, C.; Kong, D.; et al. Engineering control of bacterial cellulose production using a genetic toolkit and a new celluloseproducing strain. Proc. Natl. Acad. Sci. U. S. A. 2016, 113, E3431-E3440, doi:10.1073/pnas.1522985113.

8. Kuo, C.H.; Teng, H.Y.; Lee, C.K. Knock-out of glucose dehydrogenase gene in Gluconacetobacter xylinus for bacterial cellulose production enhancement. Biotechnol. Bioprocess Eng. 2015, 20, 18-25, doi:10.1007/s12257-014-0316-x.

9. Wu, M.; Chen, W.; Hu, J.; Tian, D.; Shen, F.; Zeng, Y.; Yang, G.; Zhang, Y.; Deng, S. Valorizing kitchen waste through bacterial cellulose production towards a more sustainable biorefinery. Sci. Total Environ. 2019, 695, 133898, doi:10.1016/j.scitotenv.2019.133898.

10. Cavka, A.; Guo, X.; Tang, S.-J.J.; Winestrand, S.; Jönsson, L.J.; Hong, F. Production of bacterial cellulose and enzyme from waste fiber sludge. Biotechnol. Biofuels 2013, 6, 25, doi:10.1186/1754-68346-25.

11. Guo, X.; Cavka, A.; Jönsson, L.J.; Hong, F. Comparison of methods for detoxification of spruce hydrolysate for bacterial cellulose production. Microb. Cell Fact. 2013, 12, 93, doi:10.1186/1475-285912-93.

12. Keshk, S.; Sameshima, K. The utilization of sugar cane molasses with/without the presence of lignosulfonate for the production of bacterial cellulose. Appl. Microbiol. Biotechnol. 2006, doi:10.1007/s00253-005-0265-6.

13. Tsouko, E.; Kourmentza, C.; Ladakis, D.; Kopsahelis, N.; Mandala, I.; Papanikolaou, S.; Paloukis, F.; Alves, V.; Koutinas, A. Bacterial Cellulose Production from Industrial Waste and by-Product Streams. Int. J. Mol. Sci. 2015, 16, 14832-14849, doi:10.3390/ijms160714832.

14. Hong, F.; Guo, X.; Zhang, S.; Han, S.; Yang, G.; Jönsson, L.J. Bacterial cellulose production from cotton-based waste textiles: Enzymatic saccharification enhanced by ionic liquid pretreatment. Bioresour. Technol. 2012, 104, 503-508, doi:10.1016/j.biortech.2011.11.028.

15. Holwerda, E.K.; Hirst, K.D.; Lynd, L.R. A defined growth medium with very low background carbon for culturing Clostridium thermocellum. J. Ind. Microbiol. Biotechnol. 2012, 39, 943-947, doi:10.1007/s10295-012-1091-3.

16. Yuste, L. Characterization of bacterial strains able to grow on high molecular mass residues from crude oil processing. FEMS Microbiol. Ecol. 2000, 32, 69-75, doi:10.1016/S0168-6496(00)00015-5.

17. Forng, E.R.; Anderson, S.M.; Cannon, R.E. Synthetic Medium for Acetobacter xylinum That Can Be Used for Isolation of Auxotrophic Mutants and Study of Cellulose Biosynthesis. Appl. Environ. Microbiol. 1989, 55, 1317-1319, doi:10.1128/AEM.55.5.1317-1319.1989.

18. Son, H.-J.; Kim, H.-G.; Kim, K.-K.; Kim, H.-S.; Kim, Y.-G.; Lee, S.-J. Increased production of bacterial cellulose by Acetobacter sp. V6 in synthetic media under shaking culture conditions. Bioresour. Technol. 2003, 86, 215-219, doi:10.1016/S0960-8524(02)00176-1. 
19. Mangayil, R.; Rissanen, A.J.; Pammo, A.; Guizelini, D.; Losoi, P.; Sarlin, E.; Tuukkanen, S.; Santala, V. Characterization of a novel bacterial cellulose producer for the production of eco-friendly piezoelectric-responsive films from a minimal medium containing waste carbon. Cellulose 2021, 28, 671-689, doi:10.1007/s10570-020-03551-6.

20. Santala, S.; Efimova, E.; Kivinen, V.; Larjo, A.; Aho, T.; Karp, M.; Santala, V. Improved Triacylglycerol Production in Acinetobacter baylyi ADP1 by Metabolic Engineering. Microb. Cell Fact. 2011, 10, 36, doi:10.1186/1475-2859-10-36.

21. Asai, T.; Shoda, K. THE TAXONOMY OF ACETOBACTER AND ALLIED OXIDATIVE BACTERIA. J. Gen. Appl. Microbiol. 1958, 4, 289-311, doi:10.2323/jgam.4.289.

22. Altschul, S. Basic Local Alignment Search Tool. J. Mol. Biol. 1990, 215, 403-410, doi:10.1006/jmbi.1990.9999.

23. Thompson, J.D.; Higgins, D.G.; Gibson, T.J. CLUSTAL W: Improving the sensitivity of progressive multiple sequence alignment through sequence weighting, position-specific gap penalties and weight matrix choice. Nucleic Acids Res. 1994, doi:10.1093/nar/22.22.4673.

24. Kumar, S.; Stecher, G.; Li, M.; Knyaz, C.; Tamura, K. MEGA X: Molecular Evolutionary Genetics Analysis across Computing Platforms. Mol. Biol. Evol. 2018, 35, 1547-1549, doi:10.1093/molbev/msy096.

25. Luo, J.; Efimova, E.; Losoi, P.; Santala, V.; Santala, S. Wax ester production in nitrogen-rich conditions by metabolically engineered Acinetobacter baylyi ADP1. Metab. Eng. Commun. 2020, 10, e00128, doi:10.1016/j.mec.2020.e00128.

26. Salmela, M.; Lehtinen, T.; Efimova, E.; Santala, S.; Mangayil, R. Metabolic pairing of aerobic and anaerobic production in a one-pot batch cultivation. Biotechnol. Biofuels 2018, 11, 187, doi:10.1186/s13068-018-1186-9.

27. Masuko, T.; Minami, A.; Iwasaki, N.; Majima, T.; Nishimura, S.I.; Lee, Y.C. Carbohydrate analysis by a phenol-sulfuric acid method in microplate format. Anal. Biochem. 2005, 339, 69-72, doi:10.1016/j.ab.2004.12.001.

28. Vazquez, A.; Foresti, M.L.; Cerrutti, P.; Galvagno, M. Bacterial Cellulose from Simple and Low Cost Production Media by Gluconacetobacter xylinus. J. Polym. Environ. 2013, 21, 545-554, doi:10.1007/s10924-012-0541-3.

29. Park, S.; Baker, J.O.; Himmel, M.E.; Parilla, P.A.; Johnson, D.K. Cellulose crystallinity index: measurement techniques and their impact on interpreting cellulase performance. Biotechnol. Biofuels 2010, 3, 10, doi:10.1186/1754-6834-3-10.

30. Bolger, A.M.; Lohse, M.; Usadel, B. Trimmomatic: a flexible trimmer for Illumina sequence data. Bioinformatics 2014, 30, 2114-2120, doi:10.1093/bioinformatics/btu170.

31. Bankevich, A.; Nurk, S.; Antipov, D.; Gurevich, A.A.; Dvorkin, M.; Kulikov, A.S.; Lesin, V.M.; Nikolenko, S.I.; Pham, S.; Prjibelski, A.D.; et al. SPAdes: A New Genome Assembly Algorithm and Its Applications to Single-Cell Sequencing. J. Comput. Biol. 2012, 19, 455-477, doi:10.1089/cmb.2012.0021.

32. Dayarian, A.; Michael, T.P.; Sengupta, A.M. SOPRA: Scaffolding algorithm for paired reads via statistical optimization. BMC Bioinformatics 2010, doi:10.1186/1471-2105-11-345.

33. Gurevich, A.; Saveliev, V.; Vyahhi, N.; Tesler, G. QUAST: quality assessment tool for genome assemblies. Bioinformatics 2013, 29, 1072-1075, doi:10.1093/bioinformatics/btt086.

34. Guizelini, D.; Raittz, R.T.; Cruz, L.M.; Souza, E.M.; Steffens, M.B.R.; Pedrosa, F.O. GFinisher: a new strategy to refine and finish bacterial genome assemblies. Sci. Rep. 2016, 6, 34963, doi:10.1038/srep34963.

35. Seemann, T. Prokka: rapid prokaryotic genome annotation. Bioinformatics 2014, 30, 2068-2069, doi:10.1093/bioinformatics/btu153.

36. Parks, D.H.; Imelfort, M.; Skennerton, C.T.; Hugenholtz, P.; Tyson, G.W. CheckM: assessing the 
quality of microbial genomes recovered from isolates, single cells, and metagenomes. Genome Res. 2015, 25, 1043-1055, doi:10.1101/gr.186072.114.

37. Arkin, A.P.; Cottingham, R.W.; Henry, C.S.; Harris, N.L.; Stevens, R.L.; Maslov, S.; Dehal, P.; Ware, D.; Perez, F.; Canon, S.; et al. KBase: The United States Department of Energy Systems Biology Knowledgebase. Nat. Biotechnol. 2018, 36, 566-569, doi:10.1038/nbt.4163.

38. Moriya, Y.; Itoh, M.; Okuda, S.; Yoshizawa, A.C.; Kanehisa, M. KAAS: an automatic genome annotation and pathway reconstruction server. Nucleic Acids Res. 2007, 35, W182-W185, doi:10.1093/nar/gkm321.

39. Okonechnikov, K.; Golosova, O.; Fursov, M. Unipro UGENE: a unified bioinformatics toolkit. Bioinformatics 2012, 28, 1166-1167, doi:10.1093/bioinformatics/bts091.

40. Zdobnov, E.M.; Apweiler, R. InterProScan - an integration platform for the signature-recognition methods in InterPro. Bioinformatics 2001, 17, 847-848, doi:10.1093/bioinformatics/17.9.847.

41. Marchler-Bauer, A.; Derbyshire, M.K.; Gonzales, N.R.; Lu, S.; Chitsaz, F.; Geer, L.Y.; Geer, R.C.; He, J.; Gwadz, M.; Hurwitz, D.I.; et al. CDD: NCBI's conserved domain database. Nucleic Acids Res. 2015, 43, D222-D226, doi:10.1093/nar/gku1221.

42. Antipov, D.; Hartwick, N.; Shen, M.; Raiko, M.; Lapidus, A.; Pevzner, P.A. plasmidSPAdes: assembling plasmids from whole genome sequencing data. Bioinformatics 2016, 32, 3380-3387, doi:10.1093/bioinformatics/btw493.

43. Rozov, R.; Brown Kav, A.; Bogumil, D.; Shterzer, N.; Halperin, E.; Mizrahi, I.; Shamir, R. Recycler: an algorithm for detecting plasmids from de novo assembly graphs. Bioinformatics 2016, btw651, doi:10.1093/bioinformatics/btw651.

44. $\quad$ Langmead, B.; Salzberg, S.L. Fast gapped-read alignment with Bowtie 2. Nat. Methods 2012, 9, 357359, doi:10.1038/nmeth.1923.

45. Gao, F.; Zhang, C.-T. Ori-Finder: A web-based system for finding oriC s in unannotated bacterial genomes. BMC Bioinformatics 2008, 9, 79, doi:10.1186/1471-2105-9-79.

46. Lagesen, K.; Hallin, P.; Rødland, E.A.; Stærfeldt, H.H.; Rognes, T.; Ussery, D.W. RNAmmer: Consistent and rapid annotation of ribosomal RNA genes. Nucleic Acids Res. 2007, doi:10.1093/nar/gkm160.

47. Lowe, T.M.; Chan, P.P. tRNAscan-SE On-line: integrating search and context for analysis of transfer RNA genes. Nucleic Acids Res. 2016, 44, W54-W57, doi:10.1093/nar/gkw413.

48. Cros, M.J.; De Monte, A.; Mariette, J.; Bardou, P.; Grenier-Boley, B.; Gautheret, D.; Touzet, H.; Gaspin, C. RNAspace.org: An integrated environment for the prediction, annotation, and analysis of ncRNA. RNA 2011, 17, 1947-1956, doi:10.1261/rna.2844911.

49. Marič, L.; Cleenwerck, I.; Accetto, T.; Vandamme, P.; Trček, J. Description of komagataeibacter melaceti sp. Nov. and komagataeibacter melomenusus sp. nov. isolated from apple cider vinegar. Microorganisms 2020, 8, 1-15, doi:10.3390/microorganisms8081178.

50. Semjonovs, P.; Ruklisha, M.; Paegle, L.; Saka, M.; Treimane, R.; Skute, M.; Rozenberga, L.; Vikele, L.; Sabovics, M.; Cleenwerck, I. Cellulose synthesis by Komagataeibacter rhaeticus strain P 1463 isolated from Kombucha. Appl. Microbiol. Biotechnol. 2017, 101, 1003-1012, doi:10.1007/s00253-0167761-8.

51. Lu, T.; Gao, H.; Liao, B.; Wu, J.; Zhang, W.; Huang, J.; Liu, M.; Huang, J.; Chang, Z.; Jin, M.; et al. Characterization and optimization of production of bacterial cellulose from strain CGMCC 17276 based on whole-genome analysis. Carbohydr. Polym. 2020, 232, 115788, doi:10.1016/j.carbpol.2019.115788.

52. Liu, M.; Liu, L.; Jia, S.; Li, S.; Zou, Y.; Zhong, C. Complete genome analysis of Gluconacetobacter xylinus CGMCC 2955 for elucidating bacterial cellulose biosynthesis and metabolic regulation. Sci. Rep. 2018, 8, 6266, doi:10.1038/s41598-018-24559-w.

53. Zhang, H.; Xu, X.; Chen, X.; Yuan, F.; Sun, B.; Xu, Y.; Yang, J.; Sun, D. Complete genome sequence 
of the cellulose-producing strain Komagataeibacter nataicola RZS01. Sci. Rep. 2017, 7, 4431, doi:10.1038/s41598-017-04589-6.

54. Jung, H. Il; Lee, O.M.; Jeong, J.H.; Jeon, Y.D.; Park, K.H.; Kim, H.S.; An, W.G.; Son, H.J. Production and characterization of cellulose by acetobacter sp. V6 using a cost-effective molasses-corn steep liquor medium. Appl. Biochem. Biotechnol. 2010, doi:10.1007/s12010-009-8759-9.

55. Wang, S.-S.S.; Han, Y.-H.H.; Chen, J.-L.L.; Zhang, D.-C.C.; Shi, X.-X.X.; Ye, Y.-X.X.; Chen, D.-L.L.; Li, M. Insights into Bacterial Cellulose Biosynthesis from Different Carbon Sources and the Associated Biochemical Transformation Pathways in Komagataeibacter sp. W1. Polymers (Basel). 2018, 10, 963, doi:10.3390/polym10090963.

56. Ramana, K. V.; Tomar, A.; Singh, L. Effect of various carbon and nitrogen sources on cellulose synthesis by acetobacter xylinum. World J. Microbiol. Biotechnol. 2000, doi:10.1023/A:1008958014270.

57. Soemphol, W.; Hongsachart, P.; Tanamool, V. Production and characterization of bacterial cellulose produced from agricultural by-product by Gluconacetobacter strains. Mater. Today Proc. 2018, 5, 11159-11168, doi:10.1016/j.matpr.2018.01.036.

58. Kuo, C.H.; Chen, J.H.; Liou, B.K.; Lee, C.K. Utilization of acetate buffer to improve bacterial cellulose production by Gluconacetobacter xylinus. Food Hydrocoll. 2014.

59. de Souza, S.S.; Berti, F. V.; de Oliveira, K.P. V.; Pittella, C.Q.P.; de Castro, J. V.; Pelissari, C.; Rambo, C.R.; Porto, L.M. Nanocellulose biosynthesis by Komagataeibacter hansenii in a defined minimal culture medium. Cellulose 2019, 26, 1641-1655, doi:10.1007/s10570-018-2178-4.

60. Jacek, P.; Dourado, F.; Gama, M.; Bielecki, S. Molecular aspects of bacterial nanocellulose biosynthesis. Microb. Biotechnol. 2019, 1751-7915.13386, doi:10.1111/1751-7915.13386.

61. Ross, P.; Mayer, R.; Benziman, M. Cellulose biosynthesis and function in bacteria. Microbiol. Rev. 1991, 55, 35-58, doi:10.1128/MMBR.55.1.35-58.1991.

62. Zhong, C. Industrial-Scale Production and Applications of Bacterial Cellulose. Front. Bioeng. Biotechnol. 2020, 8, 605374 .

63. Son, H.-J.; Heo, M.-S.; Kim, Y.-G.; Lee, S.-J. Optimization of fermentation conditions for the production of bacterial cellulose by a newly isolated Acetobacter sp.A9 in shaking cultures. Biotechnol. Appl. Biochem. 2001, 33, 1, doi:10.1042/BA20000065.

64. Velásquez-Riaño, M.; Bojacá, V. Production of bacterial cellulose from alternative low-cost substrates. Cellulose 2017, 24, 2677-2698.

65. Cao, Y.; Lu, S.; Yang, Y. Production of bacterial cellulose from byproduct of citrus juice processing (citrus pulp) by Gluconacetobacter hansenii. Cellulose 2018, 25, 6977-6988, doi:10.1007/s10570-0182056-0.

66. Kongruang, S. Bacterial cellulose production by Acetobacter xylinum strains from agricultural waste products. Appl. Biochem. Biotechnol. 2008, 148, 245-256, doi:10.1007/s12010-007-8119-6.

67. Sauer, U.; Eikmanns, B.J. The PEP-pyruvate-oxaloacetate node as the switch point for carbon flux distribution in bacteria: We dedicate this paper to Rudolf $\mathrm{K}$. Thauer, Director of the Max-PlanckInstitute for Terrestrial Microbiology in Marburg, Germany, on the occasion of his 65th. FEMS Microbiol. Rev. 2005, 29, 765-794, doi:10.1016/j.femsre.2004.11.002.

68. Kuo, C.-H.; Chen, J.-H.; Liou, B.-K.; Lee, C.-K. Utilization of acetate buffer to improve bacterial cellulose production by Gluconacetobacter xylinus. Food Hydrocoll. 2016, 53, 98-103, doi:10.1016/j.foodhyd.2014.12.034. 